\title{
XIX.
}

\section{Die normalen Bewegungen der Pharyngealmündung der Eustachi'schen Rőhre}

\author{
von \\ E. Zaufal. \\ Zweiter Artikel. \\ (Hierzu Tafel VIII u. IX.)
}

Seit der Abfassung des im Bande IX. 3. Heft dieses Archivs ersehienenen Artikels über die normalen Bewegungen der Pharyngealmundung der Eustachi'schen Röhre kam mir noch eine grössere Anzahl von Fällen (10) zur Beobachtung, die mir das in den ersten zwei Fällen Beobachtete theils bestätigten, theils hinreichend Gelegenheit boten, dașselbe noch zu ergänzen.

Zugleich bin ich in der Lage, die ersten Bilder der Pharyngealmiindung der Tuba, aufgenommen bei der Besichtigung durch die Nase, vorzulegen. Manche der in dem früheren Aufsatze vorkommenden Ausdruicke, wie Entfaltung des Bodens der Pharyngealmuindung, Eröffnung derselben ete, erhalten dadurch ibre richtige Deutung, indem sie sich zunächst nur auf Veränderungen der Ansicht der Ruhelage beziehen, ohne Rücksicht darauf, wie gross die Raumveränderungen in Wirklichkeit sind. Denn es hat die Wurdigung insbesondere der Tiefendimensionen d. i. der Entfernungen in der Sagittalebene immer darauf Ruicksicht zu nehmen, dass wir dieselben rom Standpunkte des Beobachters aus verkürzt sehen müssen, da wir das Ostium pharyngeum der Tuba mit den angrenzenden Theilen der äusseren Wand des Cavum pharyngo-nasale nicht in der Fläche, sondern mehr im Profile oder eigentlich nahezu im dreiviertel Profile ïbersehen, welcher Situation auch die Bilder entspreehen.

Ich liess das Bild in den Rahmen der Choane, der ihm 
naturgemäss znkommt, einfügen, so dass man schon aus der Form desselben die entsprechende Körperseite entnehmen kann. Ausbauchungen oder leistenartige Erhöhungen der Nasenscheidewand (senkrechter Rand des Rahmens) erscheinen auf letzteren projicirt (Eh Tf. VIII. Fig. 1, 2); dasselbe hätte auch mit einem etwa vorhandenen Rudimente der unteren Nasenmuschel zu geschehen. Um jedoch das Bild nicht unnöthiger Weise complicirt zu machen, vermied ich es und liess das Rudiment der unteren Nasenmuschel blos als leichte Erhöhung an der convexen Seite des Rahmens andeuten ( $R m$ Tf. VIII. Fig. 1. 2).

Tafel VIII. Fig. 1 und 2 gibt zwei halbschematische Figuren, von denen Fig. 1 das Bild der Ruhelage und Fig. 2 das Ostium pharyngeum in Bewegung $\mathrm{u}$. z. annähernd eine Mittellage zwischen Phonation und Schlingbewegung darstellt ${ }^{1}$ ). Tafel IX. illustrirt in natürlicher Grösse das Bild des Ostium pharyngeum dreier Falle A., B., C.

Das Bild der Ruhelage bleibt sich im Allgemeinen constant, wie ich es bereits (1. c.) beschrieben habe nnd wie es Taf. II. die Figuren 3, 5, 7, 9, 10 darstellen.

Der Tubenwulst (Tw) ist mehr oder weniger stark hervorragend, glatt, abgerundet und sein unteres Ende verbirgt sich hinter der unteren Partie der Hakenfalte (Hkf), so dass es, da die hintere Hälfte des weicken Gaumens nicht in Sicht ist, den Anschein gewinnt, als schwebe der Wulst in der Luft (Fig. 3, 5, 9, 10). Nur einmal konnte ich ihn mit Hülfe eines langen, dünnen, stark nach unten und hinten gesenkten Trichters bis herab zur oberen Fläche des weichen Gaumens verfolgen und auch eine Andeutung seiner Falte sehen.

Er verläuft entweder senkrecht nach abwärts (Fig. 9 u. 10) oder in einem nach hinten leicht convexen Bogen (Fig. 3 u. 7), erscheint sehr lang (Fig. 9, 10) oder auffallend kurz (Fig. 7), von kräftiger gedrungener Form oder mehr schlaff herabhängend.

Seine hak enförmige Umbiegung (Hk) ist meist seharf hervortretend und geht allmählich in die äussere und obere Wand des Cavum pharyngo-nasale über.

Die Hakenfalte (Hhf) ist immer vorhanden, nach vorne entweder scharf abgegrenzt oder obne dentliche Grenzen in die Schleimhaut der Choane sich verlierend. Sie zieht gewöhnlich

1) In beiden Figuren ist der Abstand der höchsten Wölbung des Hakens von der oberen Rachenwand zu hoch gezeichnet. 
in Form einer in der oberen Partie mehr senkrechten, sich danm im Bogen medianwärts krümmenden Crista nach ab- und einwärts und verbreitert sich bei ihrem Uebergange in die obere Fläche des weichen Ganmens (Fig. 8, 9, 10), sie ist blassgelblich gefärbt, glatt, wenn nicht pathologische Processe, z. B. Narben in einem beobachteten Fall von Syphilis, oder event. katarrhalische Schwellung diese Eigenschaften alieniren.

Die Tubenmündung (To) erscheint entweder als ein mebr linearer Spalt (Fig. 3, 7. 9) oder als eine 3-4 Mm. weite Oeffnung (Fig. 5, 10), die nach lebhaften, langdauernden Bewegungen, z. B. nach Declamiren, zuweilen mit schaumiger Flüssigkeit verlegt ist.

Von der hinteren Rachenwand grenzt sich der Wulst und die nach aus- und aufwärts ziehende Abdachung seiner hakenförmigen Umbiegung durch eine dunkle mehr weniger flach S-förmige Linie (Fig. 3, 5, 7, 9) ab, wodurch der Verlauf der Rosenmüller'schen Grube $(R g)$ und des Winkels, in welchem hintere, oberê und äussere Wand des Cavum pharyngo-nasale zusammenstossen, markirt wird.

Die grössere oder geringere Einengung des Uebersichtsterrains der hinteren und oberen Wand des Nasen-Rachenraums ist hauptsächlich durch vorhandene Erhöhungen an der Nasenscheidewand $(N)$ und durch Rudimente der unteren Nasenmuschel bedingt. Am besten iibersieht man diese Wände, wenn die Nasenscheidewand keine oder nur eine ganz flache Hervorbauchung besitzt und die untere Nasenmuschel vollständig fehlt (Fig. 10), während in Fig. 3 und 7 ein grosser Theil der hinteren und oberen Wand dureh Hervorragungen an der Nasenscheidewand verdeckt wird. In Fällen, wo die Form der unteren Nasenmuschel gut erhalten ist, unter ihrer Krümmung aber doch noch so viel Raum bleibt, um einen mittelstarken Trichter einzufuibren, übersieht man zuweilen den Wulst und nur wenig von der hinteren Wand; oder nur einen Theil der hinteren Wand häufig durch Lichtreflexe markirt; den Haken und den Wulst in der Ruhelage gar nicht und erst bei der Phonation und beim Schlingen erscheint der Wulst mit seiner Falte.

An der Schleimhaut der hinteren Rachenwand zeichnen sich zuweilen feine Längsfalten ab (Fig. 7, 9, 10); nicht selten sieht man auch vergrösserte Follikel, ausnahmsweise Geschwïrchen (in einem Falle rou Syphilis). 


\section{Physiologische Veränderungen der Pharyngealmündung der Tuba.}

1. Bei Respirationsbewegungen.

Am inconstantesten sind die Veränderungen, die man am Ostium bei den Athembewegungen beobachtet; nur bei ruhigem Athmen fehlte in allen von mir beobachteten Fällen constant jede darauf bezigliche Aenderung des Ostiums. - Bei forcirtem Athmen durch Nase oder Mund behält die Pharyngealmiundung entweder ihre Ruhelage, oder sie öffnet sich bei der In- oder Exspiration, um nach mehreren Athemzitgen wieder in die Ruhelage zuriickzutreten und darin zu verharren, oder der Wulst tritt bei der Exspirationsbewegung zurïck, macht bei den folgenden In- und Exspirationen kleine pendelartige Schwingungen um eine Mittellage und kehrt beim Aufhören der forcirten Respirationen sogleich in seine Ruhelage zuritick.

In den Fällen, wo ich das positive Experimentum Valsalvae machen liess, beobachtete ich constant, dass das untere Ende des Wulstes, sich scheinbar der Hakenfalte anschmiegend, nach vorne ritckte.

Beim Husten erfolgt bei jedem Exspirationsstoss mit dem Emporschnellen des weichen Gaumens ein stossweises Zurückprallen des Wulstes an die hintere Rachenwand.

\section{Bei der Phonation.}

Bei der Phonation der Vocale beobachtete ich constant in allen Fallen die bereits (1. c.) beschriebenen Veränderungen. Der Tubenwulst erscheint selbstrerständlich perspectivisch verkürzt (Fig. 4, 11, 12). Seine Falte $(W f)$ war in allen Fällen vorhanden und grenzte sich mehr oder weniger scharf von dem unteren Ende des Wulstes ab (Fig. 4, 12), war entweder sehr lang (Fig. 11) oder auffallend kurz (Fig. 8). Indem der Wulst nach rück- und einwärts rückt, macht es den Eindruck, als ob er die Schleimhaut der hinteren Rachenwand in Längsfalten, vor sich hertreibe (Fig. 8, 11, 12). Einmal sah ich, wie unmittelbar an dem zurïckgetretenen Wulste sich eine solche Längsfalte emporbauschte, so dass sie förmlich über den Wulstrand hervorquoll.

In allen beobachteten Fällen flachte sich das untere Drittheil der Hakenfalte deutlich ab.

Verschieden gestaltet sich die Flächenformation des durch 
die Phonation der Vocale zur Entfaltung gebrachten Bodens des Ostiums je nach der Verschiedenheit des Vocales und der Intensität seiner Anlautung. Bei manchen Individuen ist die Excursionsbreite grösser bei der Aussprache des Vocales a, wie im Fall C., bei anderen bei Aussprache des Vosales e, wie im Falle A., und dem entsprechend auch die Entfaltung des Bodens der Pharyngealmündung.

Bei schwachem Anschlag des betreffenden Vocales ist der Boden eben oder selbst leicht muldenförmig vertieft und von vor- nach rückwärts verkürzt. Mit der wachsenden Stärke des Anschlages wächst die Excursionsbreite des Wulstes und bildet sich, etwas mehr in der hinteren Hälfte des Bodens, ein gegen das Tubalumen nach aus- und aufwärts sich verlierender flacher Längswulst, der medianwärts sich verbreiternd in der oberen Fläche des weichen Gaumens verśchwindet. Nach vorne zu geht er in eine muldenförmige Vertiefung über, in die das untere abgeflachte Ende der Hakenfalte mit einbezogen ist, und nach rïekwärts in die schon früher (1. e.) beschriebene scharfkantige Rinne. Der Boden wird dadurch wellenförmig, nach vorne tällt das Wellenthal, nach rüekwärts der Wellenberg, an den sich dann bis zum Wulste und seiner Falte wieder ein enges Wellenthat anschliesst.

Für die Bezeichnung dieses Wulstes möchten wir den Namen Bodenwulst oder Levatorwulst $(L w)$ in Vorschlag bringen. Er ist constant und nur einmal (Fig. 11) fehlte er, selbst bei stärkerem Anschlag, and bildete sich blos ein breites, muldenförmiges Wellenthal aus.

Beim Singen gehen die Veränderungen des Ostiums in ähnlicher Weise vor sich, wie bei der Phonation.

3. Veränderungen beim Schlingacte.

Auch beim Schlingen fand ich die bereits beschriebenen Erscheinungen in allen Fällen bestätigt. Wie bei der Phonation tritt auch hier der Levatorwulst hinter der vorderen flach muldenförmigen Vertiefung des Bodens, jedoch noch markirter, in die Erscheinung, selbst da, wo er bei der Phonation fehlte (Fig. 13, cf. 11). Der Levatorwulst fullt den Rahmen des Ostium nicht aus, nur unter pathologischen Verhältnissen, bei Hypertrophie der Tonsille, sah ich ihn während des Sehlingens bis zum Haken hinauf gedrängt werden. Manchmal machen sich auf der Axe des Levatorwulstes senkreeht gestellte, nahe aneinander 
liegende, halbringförmige Zeichnungen bemerkbar, die dem blassgelblichen Wulste das Aussehen einer Vogeltrachea geben.

4. Die Bewegungen bei starkem Senken des Unterkiefers und Hervorschnellen der Zunge

sind inconstant. In einigen Fällen sind sie, wie (l. c.) beschrieben, deutlich ausgeprägt, während ich sie in anderen Fällen vermisste.

Auch die Zwangsbewegungen beim Niederhalten des Zungengrundes und bei gleichzeitiger Phonation fand ich nicht in allen Fällen. Einen ausgezeichneten Fall illustrirt Fig. 14.

Prag, 28. December 1874.

Erklärung der Abbildungen (TafeI VIII a. IX).

Fig. 1 u. 2. Halbsehematische Figuren der Pharyngealmündung der Tuba in der Rahelage und in Bewegung.

$N$ Nasenscheidewand. - Eh Erhöhung an derselben. Cho Choane. - $R m$ Rudiment der unteren Nasenmuschel. $H p h$ Hintere Pharynxwand. - Rg Rosenmuller'sche Grube. Tw Tubenwulst. - To Tubenostium. - Hk Haken. - Hkf Hakenfalte. - Wf Wulstfalte. - $L w$ Levatorwnlst.

$A, A, A, A$ Bilder des Ostium ph. t. desselben Individnums und zwar: Fig. 3 rechtes Ostium in der Ruhelage. - Fig. 4 rechtes Ostium bei der Phonation des Vocales e. - Fig. 5 linkes Ostium in der Ruhelage. - Fig. 6 linkes Ostium beim Schlingen. - $B B$ von einem anderen Individunm. - Fig. 7 reehtes Ostinm in der Ruhelage. - Fig. 8 reehtes Ostium bei der Phonation des Vocales a. - $C, C, C, C, C, C$ von einem dritten Individuum. - Fig. 9 rechtes Ostium in der Ruhelage. - Fig. 10 linkes Ostium in der Ruhelage. - Fig. 11 rechtes Ostium bei der Phonation des Vocales a. Fig. 12 linkes Ostium bei der Phonation des Vocales a. - Fig. 13 rechtes Ostinm beim Schlingen. - Fig. 14 rechtes Ostium beim Niederdrücken des Zungengrundes mit gleichzeitiger Phonation des Vocales $a$. 

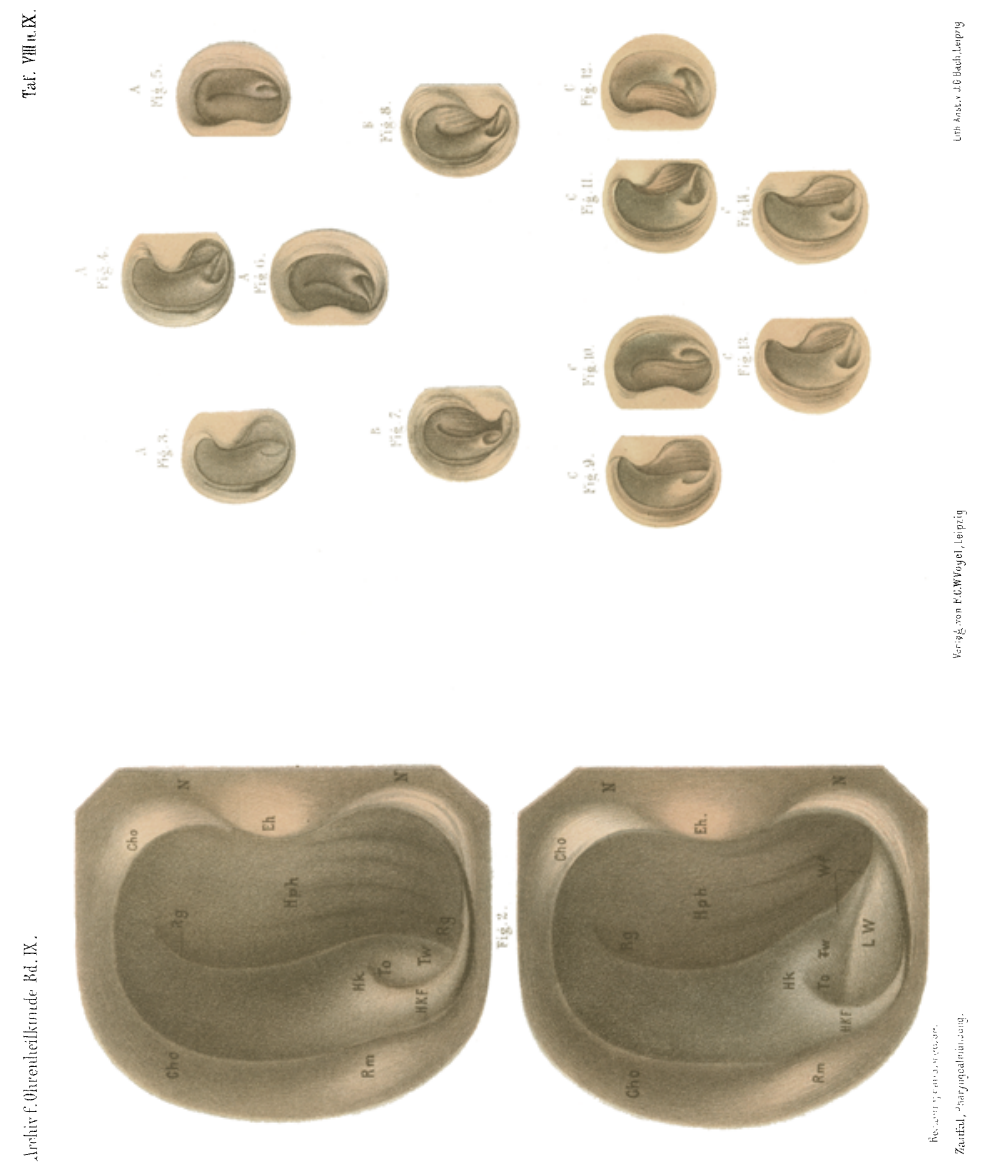